\title{
Face to face with COVID-19: highlights of challenges encountered in various ENT practices across the Czech Republic (a national survey)
}

\author{
Anasuya Guha ${ }^{1}$ (D) Jan Plzak ${ }^{2} \cdot$ Martin Chovanec $^{1}$
}

Received: 8 June 2020 / Accepted: 6 August 2020 / Published online: 19 August 2020

c) Springer-Verlag GmbH Germany, part of Springer Nature 2020

\begin{abstract}
The SARS-CoV-2 virus causing the COVID-19 disease created considerable complications and disruptions due to it's pandemic spread. The nature of the disease left certain clinical specialties more vulnerable than others, causing serious problems with organization of care of patients. Most studies concentrated on protocols and strategies to control the situation and reduce further spread amongst medical fields. Only few were designed to identify the various issues faced by doctors. We decided to design a questionnaire addressing relevant matters for doctors working in the field of ear, nose and throat (ENT). This was then sent to all the members of the Czech Society of Otorhinolaryngology. All ENT doctors were invited to fill out the survey within a stipulated time. The aim of the study was to evaluate the impact of a pandemic situation amongst such a high risk specialty. Our results showed a significant difference between the first cases of COVID-19 and progress of the disease during April 2020 in terms of workplace preparation, adequacy of personal protective equipment and other disposable aids. There was also inadequate support of medical personnel within the ENT practices. Furthermore, financial losses were also experienced due to mainly patient-related problems. In conclusion, the obtained results were used to formulate some recommendations for the public, patients, medical staff and employers. These suggestions will hopefully help avoid a repetition of the glitches if faced with another pandemic situation.
\end{abstract}

Keywords COVID-19 $\cdot$ Pandemic $\cdot$ ENT $\cdot$ PPE $\cdot$ Disposable aids $\cdot$ Financial loss

\section{Introduction}

A pandemic situation although a rare occurrence, cannot be considered an alien phenomenon. Needless to say, the burden of a pandemic disease relates to problems encountered immediately during the situation and continues further to a period of time when the situation stabilizes within a defined environment and subsequently globally. However, the eradication of such rampant disease is usually impossible, so the main goal is keeping the situation under control. This time,

Anasuya Guha

anasuya.guha@fnkv.cz

1 Department of Otorhinolaryngology, Charles University, 3rd Faculty of Medicine and University Hospital Kralovske Vinohrady, Srobarova 1150/50, Prague 10034, Czech Republic

2 Department of Otorhinolaryngology, Head and Neck Surgery, Charles University, 1st Faculty of Medicine and University Hospital Motol, V Uvalu 84/1, Prague 15006, Czech Republic the end of the year 2019, brought with it, a nightmare, the SARS-CoV-2 virus (classified by WHO) [1-4] that causes the Corona Virus Disease-19 (COVID-19). This originated from China and spread across 213 countries and territories including 2 international conveyances over a period of 6 months, thus affecting approximately 4,5 million people [2].

Czech Republic encountered the virus on the 1st of March 2020 with the first 3 new cases. A state of emergency was declared across the country on the 12th March with lockdown measures. In a span of 2 months, more than 8000 cases were reported across the country.

Following the course and the spread of the disease, it soon became evident, that the likelihood of acquiring COVID-19 from contaminated products and surfaces or infected patients is high amongst certain specialities than others. We could thereby conclude that primarily those working in the field of the head and neck (ear, nose and throat, oral and maxillofacial surgeons, dentists, ophthalmologists and so on), general practitioners and intensive care physicians/anaesthesiologists are the most vulnerable. Therefore, we decided 
to design a national survey for ear, nose and throat (ENT) doctors to analyse certain important parameters related to clinical practice.

In ENT clinical settings, this brought about an enormous influx of unexpected problems. These included inexperience and shortage of time in handling the ferocity of the disease across various health institutes, redefining and revising safety protocols for staff and patients [5-13], deficit in personal protective equipment (PPE) [14], shortage of sterilization and disinfections products, reorganizing departments with provision of isolation for COVID-19 suspected and positive patients, replacement of unavailable staff as well as support for medical personnel vulnerable, contaminated or in personal contact with infected patients [15-17]. Finally a certain extent of economic and financial instability due to reduction in clinical times and operating lists, physical exhaustion and psychological burden from workplace [18] including quarantine acquired stress amongst staff were also observed. As far as we were aware of, only 1 survey directly involved the participation of doctors practicing in the region of the head and neck (oral and maxillofacial surgery, plastic and reconstructive surgery, ENT, neurosurgery, ophthalmology and so on). It was a worldwide study conducted by AO CMF Davos (Switzerland) for all their members. Their questionnaire focussed on guidelines followed by doctors, adequacy and provision of PPE by hospitals and if elective surgery has been continued. Taking into consideration all these factors and the lack of substantial studies reflecting the impact of COVID-19 specifically in ENT practices, we formulated a questionnaire to address certain important issues. This was to determine the response of ENT doctors to the COVID-19 situation in a clinical setting, identify the deficits and problems faced by the ENT doctors in their daily practices, re-evaluate the overall risk in their individual practices and also finally assess the level of personal and financial loss amongst ENT practitioners.

\section{Materials and methods}

This study was carried out in April 2020. Target population was doctors practicing Otorhinolaryngology in Czech Republic. A survey questionnaire using Google forms was designed to study the responses of ENT surgeons when coming face to face with the COVID-19 situation. It contained a mixture of 17 multiple choice questions that were mandatory and 1 optional question regarding the name of the medical practice. One out of the seventeen questions had the option of adding further comments. This was translated into Czech language. It was then sent via email and sms with an online link to the survey with the help of the Czech Society of Otorhinolaryngology and Head and Neck Surgery. A total of 900 ENT doctors were invited to complete the survey. Online access to the questionnaire was available from 15th April 2020 to 26th April 2020. Data compilation and analysis were done using Microsoft Excel.

\section{Results}

One hundred and eighty-one ENT practitioners filled in the survey completely, comprising of about $63.5 \%$ female doctors and $36.5 \%$ male doctors. Ninety-six doctors work in hospital settings and 85 in private practices. $32.6 \%$ of all doctors had 21-35 years of practice in this field. Risk level of contracting COVID-19 within the ENT specialty was perceived as high by 83 doctors and very high by 63 doctors.

A change in pattern was observed with respect to preparation of workplace to manage crisis at the time of first recorded cases of COVID-19 infection and current situation in April 2020 in Czech Republic (Fig. 1).
Fig. 1 Comparison of workplace preparation during the 1 st recorded cases of COVID-19 and current situation

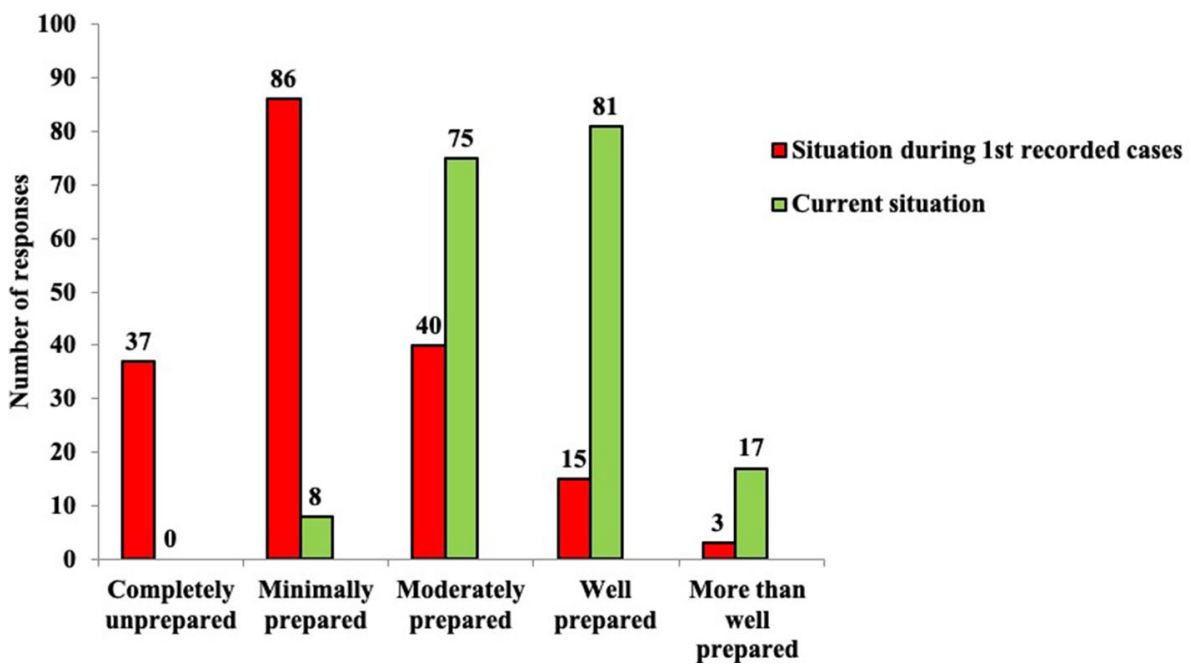


The same pattern was seen with regard to personal protective equipment (PPE) to ensure safe ENT care to prevent infection and spread of COVID-19 (Fig. 2).

In response to the question regarding the items deficient at your current practice, doctors were allowed to select as many as applicable. Out of 181 doctors, 18 selected disinfection and sterilization products, 57 had issues with PPE (respirators, shield, goggles, etc.), 75 were short of disposable aids (protective coats, gloves, surgical instruments, gowns, etc.), 15 were deficit of all of the above and in contrast 53 doctors reported no deficits.

With regards to other problems faced in the clinical practice, respondents were also allowed to select more than one option; most of them had patient-related problems (Fig. 3).

An alarming $42.5 \%$ of the practitioners were unaware of any support being provided by the individual establishments in case an employee contracts COVID- 19.

About 37 individual comments were entered as other problems encountered during the situation, most were concerned with the economic and financial problems, some feel they were ill-informed of the situation and lack adequate support from their respective employers; in certain hospital settings, sudden deployment in COVID-19 units has been unpleasant and the rest are concerned about the quality of care of patients with minimal co-operation from other medical units. Here, ENT surgeons have also suggested the issuing of public statements about health safety and re-iteration of lockdown principles to reduce the influx of unnecessary patients and risk of contamination. A few extracts from individual responses are stated here.

Example 1-from hospital practice

"completely reversed biorhythm - we exchanged to the red (COVID) zone, in addition to the consultation in the green (NON-COVID) zone, we practically do not have 2 days off in a row or only exceptionally. Chronic hypoxia when using a respirator after 5 h, headache; skin problems from permanent wearing of 2 gloves, in the face from the respirator; in addition, a problem with dioptric glasses and discomfort from other protection aids"

Example 2-from hospital practice

"dissatisfaction with the organization of care: care for COVID suspected/positive patients is adequate, but chronic and oncological patients who need medical
Fig. 2 Comparison of provision of PPE by the workplace during the 1 st recorded cases of COVID-19 and current situation

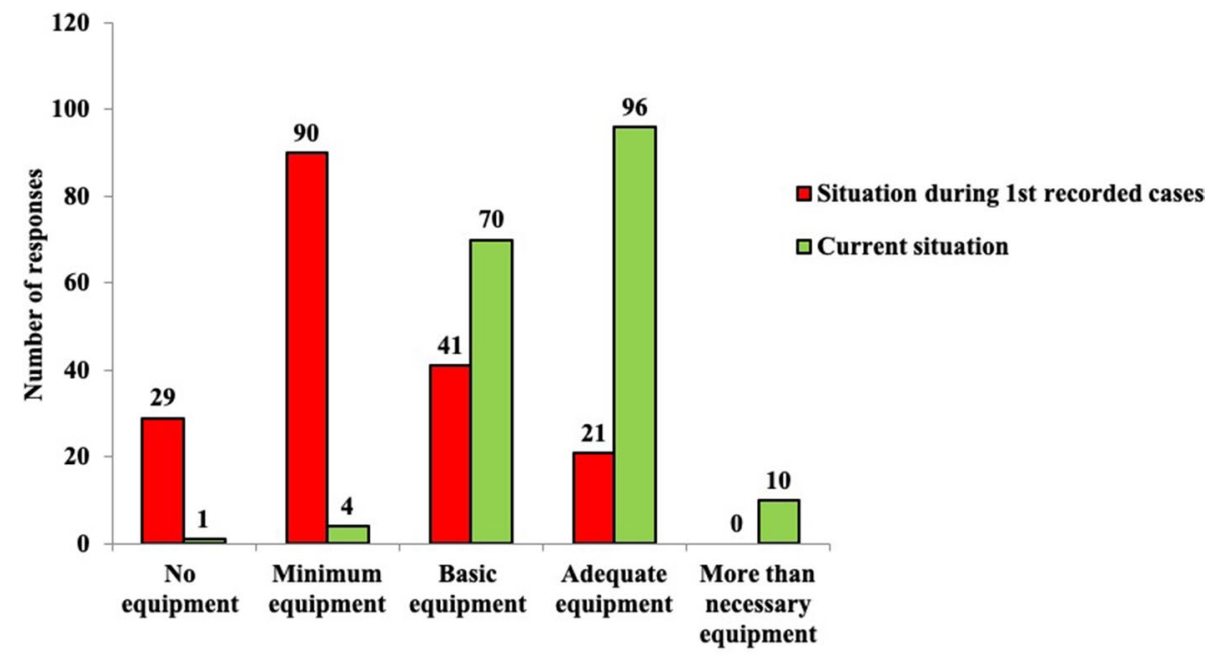

Fig. 3 Response of ENT doctors to other problems faced in their clinical practices

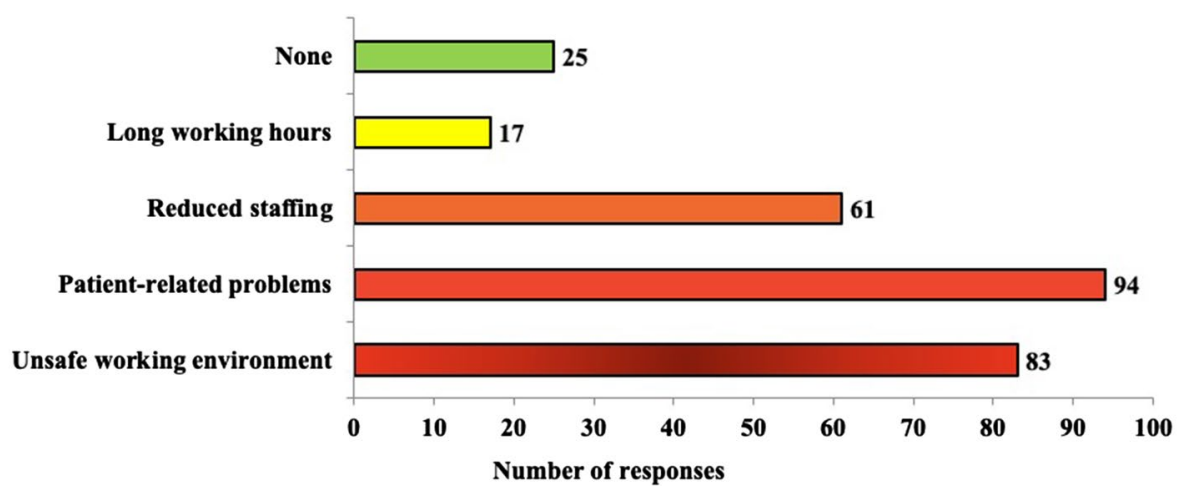


help sometimes more than "COVID" patients unfortunately do not get it"

Example 3-from a private practice

"I, myself, am in immunosuppression and still monitored for oncology, yet I worked from the beginning, despite the lack of PPE, when the import of PPE from China began, I was in bed with fever and viral infection... now I am on medical leave"

Example 4-from a private practice

"we lack FFP3 respirators - we did not receive any and are still unavailable to outpatients, the prospect of reduced income with a small number of patients. There are no algorithms for how to proceed with the gradual dismantling of care - is it possible to perform audio examinations in the cabin for asymptomatic patients? If they have COVID-19, will you not thoroughly disinfect the audio booth?"

About $50.8 \%$ of doctors agreed that all (psychological counselling, medical and financial) services should be received by ENT staff in the time of crisis.

And lastly, only 2 people thought the situation is resolved and in contrast, 108 concluded that it would resolve in a few months (Fig. 4) in response to the length of time that the major difficulties associated with the pandemic situation might last, thus interfering with the performance of the profession.

\section{Discussion}

Although, most authors discussed guidelines and protocols to ensure safe practice in relation to ENT practice [5-10, 14-16, 19, 20], a few questionnaire-based surveys have also been published [21, 22]. Interestingly, this included a study based on maxillofacial surgeons. A questionnaire with 33 questions were designed and sent worldwide. The first section was questions based on the Disease Outbreak Response
System Condition (DORSCON) scale, the second section was used to obtain a photograph of the respondent's practice before the outbreak and the third section detailed on practice situation during the outbreak. A total of 166 responses were received from 54 countries [22].

Since ear, nose and throat symptoms may precede the development of severe COVID-19, during such a pandemic, patients with sore throat, cough, dyspnoea, hyposmia/anosmia with a travel history to regions with confirmed COVID19 cases, should be considered as potential COVID-19 patients [20]. Billboards, advertisements, large readable posters outside clinics, leaflets through the door and other media should serve as strong devices in teaching people how to treat mild symptoms at home; providing emergency contact numbers regarding health advice should be mandatory. Even though the reduced number of patients has led to significant financial instability, especially in small ENT practices, the aim in such infectious pandemics is to restrain and control the situation at hand as well as reduce further spread amongst medical staff. A paper published from Italy also suggested the importance of revising the triage system as the disease evolves [11]. The same authors further mentioned the importance of separating the doctors working directly with COVID-19 patients from the rest [11], such precautions are rarely taken in hospital settings, and as our survey has shown ENT doctors working within the department, were deployed in COVID-19 units whenever required. Doctors aged 50-60 years are also at high risk and should be discouraged from working in clinical practices or COVID-19 frontline [16], $15.5 \%$ of our study group have had greater than 35 years of practice, so they do not fall into the recommended category. Elderly doctors with their own private practices would be disadvantageous in this respect.

Authors have also re-iterated the importance of PPE [11, $12,14,23$ ] and more so in Otorhinolaryngology, where, appropriate protection and the practice of disinfection along with the use of disposable instruments, where feasible, is of prime importance; thus when examining or performing a potential aerosol generating procedure or operating, the minimum PPE required is at least a filtering mask, visor (or
Fig. 4 Estimated time period that the COVID-19 related difficulties are expected to last

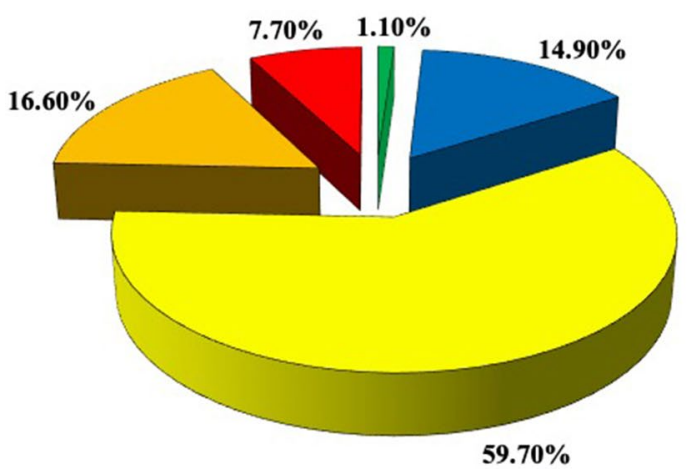

$\square$ The crisis is already over

$\square$ The difficulties will last for weeks

$\square$ The difficulties will last for months

$\square$ The difficulties will last for years

It will never be possible to return to its original state 
goggles), disposable gown and gloves. Filtering masks have been shown to effectively protect against aerosols and coronaviruses, thus the recommendation is for a mask meeting FFP3 European standards which specify a minimum $99 \%$ filtration $[14,19,20,23]$. The study from AO CMF also acknowledges the shortage of PPE across different practices [21] with variance in results amongst continents. In our survey, a comparative improvement can be seen when analysing the equipment available during the first case of COVID19 in Czech Republic and current situation; however, most private practices have not been able to afford this essentiality due to lack of funds and not all hospitals are optimally equipped due to lack of supply. As per guidelines, all nonurgent surgery should be postponed for the safety of all. Most craniomaxillofacial surgeons did stop elective surgery in the worldwide survey [21]. Individual comments received by us during the survey also shows reduction in surgery. In children, incidence of symptomatic COVID-19 (1-5\%) is low and of good prognosis [10]. No specific problems were reported by paediatric ENT practitioners in our study.

But what stands out singularly is the question of ethics, thus the patient comes first even in a pandemic situation. This includes treatment of patients during the COVID19 situation, difficulties in managing COVID-19 patients and the impact on the management of COVID-19 negative patients with other disorders $[11,12]$. This study has shown, such concerns has been raised amongst ENT surgeons and it poses a real dilemma and threat to patients who need care due to more serious illnesses.

No surveys have been conducted solely in the field of otorhinolaryngology to address the issues as seen by ENT practitioners on a daily basis during a crisis. Our study shows a representative group of 20\% amongst all ENT surgeons in Czech Republic; however, it has served the purpose of the survey. Furthermore, the worldwide survey done on doctors working in maxillofacial surgery also had a response rate of $20.2 \%$, out of 822 invitations [22].

\section{Conclusions}

Public announcements through media and clinical practices should be made available in all pandemic situations to reduce the influx of non-urgent patients and encourage seriously ill people to come forward without the fear of acquiring COVID-19 through contact with others. Instituting long-term lockdown without actually explaining the possible spread and clinical course of the disease, complications, unpredicted outcome will lead to increased risk of spread of infection and mortality.

A regularly revised triage will reduce the number of untreated patients who are seriously immunocompromised and are in need of urgent treatment. A system in the form of a written consent with legal addendums should be introduced stating the risk of incurring a stiff penalty and or legal punishment, if a patient fails to comply with the rules of the clinical practice in any way.

In hospital care, the importance of multidisciplinary management and co-operation between different specialties along with frequent feedback and discussion with hospital management is essential. Data from COVID-19 patients should be shared with the relevant authorities to keep the system updated. Every ENT workplace should uphold updated guidelines and protocols. In case of any pandemic situation, ENT staff with prior chronic illnesses or age beyond 60 years, should be encouraged to work from home and provided with certain compensation until the situation stabilizes. Every ENT practice should be provided with adequate and appropriate protective gear, failing which a medical staff may be able to decline work. All staff should be kept updated about the current situation and of any anticipated changes in the future. Changes in work pattern or environment should be addressed by a signed contract with new conditions. Maximum success can be achieved if staff are aware of the tasks they might face. Appreciation for work beyond the call of duty in the form of acceptable financial compensation will help encourage staff. Complete medical and financial support must be provided in case a staff contracts the disease.

ENT is one of the most vulnerable specialties, so strict measures should be undertaken at all times. Finally, in dire times, the importance of telehealth and thus telemedicine could provide a sufficient bridge and essential tool for the continuing care of patients.

Acknowledgements None.

Funding None.

Availability of data and material Available on requestCode availability (Software application or custom code): not applicable.

\section{Compliance with ethical standards}

Conflicts of Interest The authors declare that they have no conflict of interest.

Research involving human participants and/or animals Not applicable.

Informed consent to participate Not applicable. 


\section{References}

1. WHO (2020) Coronavirus disease (COVID-19) situation reports. https://www.who.int/emergencies/diseases/novel-coron avirus-2019/situation-reports

2. https://www.worldometers.info/coronavirus/. Accessed 20 May 2020

3. Guo YR, Cao QD, Hong ZS, Tan YY, Chen SD, Jin HJ, Tan KS, Wang DY, Yan Y (2020) The origin, transmission and clinical therapies on coronavirus disease 2019 (COVID-19) outbreak-an update on the status. Mil Med Res 7(1):11. https://doi. org/10.1186/s40779-020-00240-0

4. GOV.UK (2020) https://www.gov.uk/government/publications/ wuhan-novel-coronavirus-infection-prevention-and-control\#histo ry Accessed 19 May 2020

5. ENT UK (March 2020) Guidance for ENT during the COVID19 pandemic. https://www.entuk.org/guidance-ent-during-covid -19-pandemic

6. ENT UK (April 2020) Clinical guide to surgical prioritisation during the coronavirus pandemic. https://www.entuk.org/clini cal-guide-surgical-prioritisation-during-coronavirus-pandemicent-specific

7. Czech Society of Otorhinolaryngology and Head and Neck Surgery (2020) COVID-19 a ORL a chirurgie hlavy a krku v České republice. (Content in Czech language). https://www.otorinolar yngologie.cz/novinky/covid-19-a-orl-a-chirurgie-hlavy-a-krku-vceske-republice-377Accessed 20 May 2020

8. British Laryngological association (April 2020) COVID-19 tracheostomy guideline. https://www.britishlaryngological.org/news/ tracheostomy-guideline-covid-19

9. McGrath BA, Brenner M, Warrillow SJ et al (2020) Tracheostomy in the COVID-19 era: global and multidisciplinary guidance. Lancet Respir Med. https://doi.org/10.1016/s2213-2600(20)30230-7

10. Leboulanger N, Sagardoy T, Akkari M et al (2020) COVID-19 and ENT Pediatric otolaryngology during the COVID-19 pandemic Guidelines of the French Association of Pediatric Otorhinolaryngology (AFOP) and French Society of Otorhinolaryngology (SFORL) [published online ahead of print, 2020 Apr 18]. Eur Ann Otorhinolaryngol Head Neck Dis. https://doi.org/10.1016/j.anorl .2020.04.010

11. Simon F (2020) Ethical questions related to Covid-19 and ENT practice. Eur Ann Otorhinolaryngol Head Neck Dis. S18797296(20):30099. https://doi.org/10.1016/j.anorl.2020.04.009

12. Rosenbaum L (2020) Facing Covid-19 in Italy-ethics, logistics, and therapeutics on the epidemic's front line. N Engl J Med 382:1873-1875. https://doi.org/10.1056/nejmp2005492
13. Mitura $\mathrm{K}$ et al (2020) Guidelines for the management of surgical departments in non-uniform hospitals during the COVID-19 pandemic. Pol Przegl Chir 92(2):42-53

14. Sullivan OED (2020) PPE guidance for covid-19: be honest about resource shortages. BMJ 369:m1507. https://doi.org/10.1136/bmj. $\mathrm{m} 1507$

15. Godlee F (2020) Protect our healthcare workers. BMJ. https://doi. org/10.1136/bmj.m1324

16. Ashcroft J (2020) Keep older healthcare workers off the covid19 front line. BMJ 369:m1511. https://doi.org/10.1136/bmj. m1511

17. Yeo D, Yeo C, Kaushal S, Tan G (2020) COVID-19 \& the general surgical department-measures to reduce spread of SARS-COV-2 among surgeons. Ann Surg. https://doi.org/10.1097/sla.00000 00000003957

18. Brooks SK, Dunn R, Amlot R, Rubin GJ, Greenberg N (2018) A systematic, thematic review of social and occupational factors associated with psychological outcomes in healthcare employees during an infectious disease outbreak. J Occup Environ Med 60(3):248-257. https://doi.org/10.1097/jom.0000000000001235

19. Tysome JR, Bhutta MF (2020) COVID-19: protecting our ENT Workforce. Clin Otolaryngol 45:311-312. https://doi.org/10.1111/ coa. 13542

20. Krajewska J, Krajewski W, Zub K, Zatoński T (2020) COVID-19 in otolaryngologist practice: a review of current knowledge. Eur Arch Otorhinolaryngol 277:1885-1897. https://doi.org/10.1007/ s00405-020-05968-y

21. Tas J, Dodson T, Buchbinder D et al (2020) The global impact of COVID-19 on Craniomaxillofacial surgeons. Craniomaxillofacial Trauma Reconstruction. https://doi.org/10.1177/1943387520 929809

22. Maffia F, Fontanari V, Vellone P et al (2020) Impact of COVID19 on maxillofacial surgery: a worldwide survey. Int J Maxillofac Surg. https://doi.org/10.1016/j.ijom.2020.04.015

23. Offeddu V, Yung CF, Low MSF, Tam CC (2017) Effectiveness of masks and respirators against respiratory infections in healthcare workers: a systematic review and meta-analysis. Clin Infect Dis 65(11):1934-1942

Publisher's Note Springer Nature remains neutral with regard to jurisdictional claims in published maps and institutional affiliations. 